\title{
Magnetic measurement system of sandy gravel specimens shape during tests in a large triaxial apparatus
}

\author{
Roberta Ventini ${ }^{1, *}$, Alessandro Flora ${ }^{1}$, Stefania Lirer $^{2}$, and Claudio Mancuso ${ }^{1}$ \\ ${ }^{1}$ Federico II University, Department of Civil, Architectural and Environmental Engineering, 80125 Naples, Italy \\ ${ }^{2}$ Guglielmo Marconi University, Sustainability Engineering Department, 00193 Rome, Italy
}

\begin{abstract}
As well known, rockfill behaviour is strongly influenced by both intrinsic (mineralogy and size of particles, grain shapes, soil grading etc.) and state parameters (relative density, stress state, relative humidity). To investigate their mechanical response to stresses and relative humidity $(\mathrm{RH})$ loadings, a large size triaxial device $(\mathrm{H}=410 \mathrm{~mm}, \mathrm{D}=200 \mathrm{~mm}$ ) has been developed at the University of Naples Federico II (Italy), including modifications required to impose partially saturated conditions in the specimen by means of the vapour equilibrium technique. In order to evaluate local axial and radial strains and global volumetric strains in partially saturated conditions, a magnetic shape detector device has been designed and installed. The accuracy of this system has been evaluated in some isotropic compression triaxial tests on compacted sandygravel specimens. The experimental data clearly show the effectiveness of the magnetic system in the measurement of axial displacements while the measurement in radial direction appear to be strongly affected by the non-linearity of the complex magnetic field generated during the test and requires further checking tests.
\end{abstract}

\section{Introduction}

The mechanical characterization of coarse-grained soils has been traditionally performed by laboratory tests in large oedometer and/or triaxial devices.

Over the years, testing equipment underwent significant changes to enable a reliable description of their stress-strain behaviour in partially saturated conditions [1]. The results obtained through the new testing techniques helped to develop new constitutive models, and a deep understanding of the partial saturated soils behaviour $[2,3,4]$.

The paper describes the large triaxial cell used in the geotechnical laboratory of the University of Napoli Federico II, and the details of an innovative local magnetic system set-up in order to measure axial and radial strains. The measurements, carried out with the magnetic system, have been compared with those obtained by means of the traditional measurement system. The single test presented in this paper is representative of the general trend observed in a number of twenty tests performed up to date, highlighting limits and potentials of the new magnet system.

\section{Large triaxial cell}

The High Pressure Stress Path (HPSP) cell is a large triaxial device based on the Bishop and Wesley [5] hydraulic control of stress path. The cell is equipped with an inner submergible load cell directly placed on the top of the specimen. The triaxial cell scheme is shown in Figure 1.

The apparatus allows to test saturated and unsaturated specimens with a diameter of $200 \mathrm{~mm}$ and a height of 410 $\mathrm{mm}$. The equipment allows to apply a maximum axial load and cell pressure of $200 \mathrm{kN}$ and $2 \mathrm{MPa}$ respectively.

Separate lines of drainage are connected to the lower and upper bases of the specimen with independent lines for pore pressures and volumetric strains measurements. Taps on the mentioned lines allow the hydraulic disconnection of the drainage circuits, ensuring a high versatility in execution of different tests.

The HPSP apparatus includes an external control unit containing the electrical supply of transducers, the digital acquisition systems of the signals from the mentioned transducers and the electro-valves for the control in feedback of the water and cell pressures. The cell is finally equipped with a hydraulic power pack able to pressurise a RAM actuator up to the maximum pressure and a personal computer that runs a software for the feedback management of the tests.

Compared to a conventional triaxial cell, the HPSP differs mainly in the fact that the axial load is applied by means of a piston which pushes the soil specimen against a fixed internal load cell. The maximum piston stroke is $200 \mathrm{~mm}$ while its diameter is $60 \mathrm{~mm}$. Axial displacements are measured internally using a submerged LVDT transducer $( \pm 3 \mathrm{~mm}$ stroke, $0.3 \%$ accuracy) that is connected to the loading piston allowing the measurement of the axial strain of the specimen.

\footnotetext{
*orresponding author: roberta.ventini@unina.it
} 


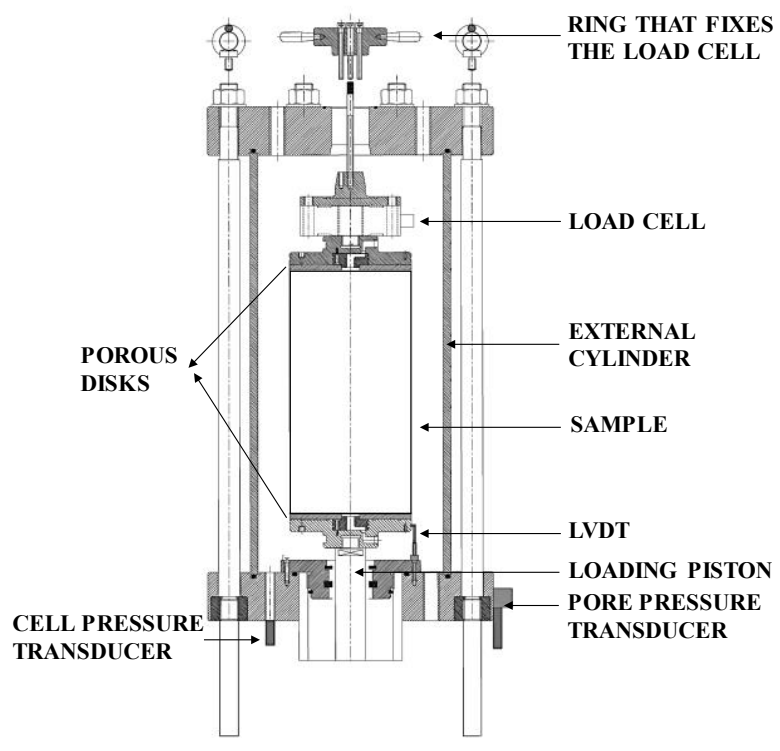

Fig. 1. Large triaxial cell scheme.

The software running the HPSP allows to perform tests under both controlled rate of loading or controlled rate of strain, as said with automatic feedback control. In the case in which the control is operated on the load, the feedback signal is provided by the load cell; instead, if the control is on the displacement, the feedback signal is provided by the LVDT transducer of the axial displacement.

The current state of the specimen is elaborated by the control system that regulates the pressures and is detected by a series of transducers, such as:

- the load cell for measuring axial load on the specimen;

- two pressure transducers (CELL and PORE) transmitting the feedback signal for cell and pore pressure control;

- a differential pressure transducer (DPT) to measure the volume changes of the specimen by the volume of water exchanged along the drainage line (tests on water saturated specimen) or by the volume of water exchanged by the cell (tests on unsaturated specimens);

- the LVDT for measurement of axial strains;

- an LVDT for measurement of the displacements of the pressure multiplier (external Volume Gauge).

It is therefore possible to perform a wide variety of tests by means of independent controls of cell, axial load and pore pressures.

In order to carry out tests in partially saturated conditions, the apparatus has been equipped with a system able to apply the vapour equilibrium technique. In particular, the triaxial cell allows to circulate air in a closed circuit between a watertight vessel containing a saturated solution of salt and water (at prescribed relative humidity), and the soil specimen, thus allowing to control, at equilibrium, the total suction in the pore air of the tested material. The whole system is located in a temperature controlled room where the maximum temperature variation is $\pm 1.5^{\circ} \mathrm{C}$.

\section{Magnetic shape detector system}

As already stated, in the HPSP cell the measurement of the volumetric strains in saturated conditions is made in a classical way by means of burettes and a differential pressure transducer (DPT). Accurate measurement of volume changes in partially saturated specimens, including changes in water and air volumes in the pores, involves greater difficulties than in saturated specimens.

Several techniques, described in the literature, allow both global and local measurements, depending on the position of the transducers. An innovative technique was developed by Romero et al. [6] and more recently by Messerklinger et al. [7], who used a laser system to measure respectively radial strains and change in volume during triaxial test.

A novel feature of the HPSP cell is the use of a magnetic system called Magnetic Shape Detector (MSD). This system was designed for the measurement of local axial and radial strains as well as for the measurement of global volume strains in partially saturated conditions.

The system consists of six N40 cylindrical magnets in neodymium, iron and boron alloy $(\mathrm{NdFeB})$, with a diameter of $10 \mathrm{~mm}$ and a height of $40 \mathrm{~mm}$ (Fig. 2b) and as many triples of Hall sensors. The magnets are glued on the latex membrane which wraps the specimen in the positions shown in Fig. 2a. The sensors are locked in fixed positions on the inner part of the external aluminium cylinder in Fig. 1, so that, at the beginning of the test, sensors' centres of gravity are perfectly aligned with the magnets' ones.

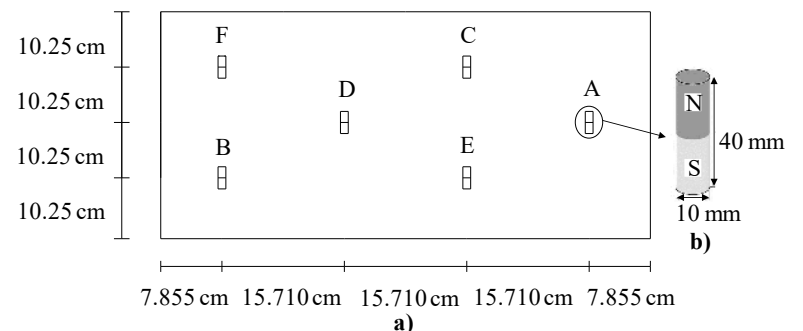

Fig. 2. Magnetic shape detector system: a) positioning scheme of the magnets on the external surface of the specimen; b) zoom of the magnet.

The sensors and the magnets mounted inside the triaxial cell are used to scan the specimen's surface during the tests. In fact, the sensors used are able to detect axial and radial displacements and rotations of the magnets placed in positions B, C, E, F (see Fig. 2a) while of the magnets $\mathrm{A}$ and $\mathrm{D}$ are able to detect only axial and radial displacements.

A data processing software allows the display of the magnets' movements during the test time thanks to the calculation of the changes in the magnetic field induced by the permanent cylindrical magnet. Based on the measured displacements a software is also able to return the variation of the side profile of the specimen by means of an interpolation method. In particular, the software mediates the position of the magnets placed at the same initial height and, given the lack of information related to the rotation of the magnets in the central positions A and 
$\mathrm{D}$, assumes vertical tangents at the centre and at the lower base of the specimen. The vertical zero position of the profile is set at the base of the upper porous stone (which is fixed), to exclude bedding errors due to the initial compliance of the axial loading system. This fixed point marks the zero value of the vertical axis measuring the position of any target along the lateral surface of the specimen, and is directed towards the lower base, while the axis measuring the horizontal displacements is directed towards the outside of the specimen.

Having noted the positions of the bases and the lateral profile, the current volume of the specimen is easily measured by an integral calculation.

\subsection{Calibration tests}

It has been demonstrated in literature [8] that the region of linear response of a magnet is only around its centre thus explaining why only a short path can be measured with a single sensor. The increased linearity error depends on the type of sensor and on the strength of the magnetic field. In order to create an accurate system, the linearity errors have been reduced by using more than two sensors, a magnet with large dimensions and a post-processing compensation such as linearization to correct residual error.

The proposed system allows linear measurement through a vertical displacement of $10 \mathrm{~mm}(0 /-10 \mathrm{~mm})$ for magnets $\mathrm{C}$ and $\mathrm{F}$, of $20 \mathrm{~mm}(15 /-5 \mathrm{~mm})$ for magnets $\mathrm{A}$ and $\mathrm{D}$, and of $30 \mathrm{~mm}( \pm 15 \mathrm{~mm})$ for magnets $\mathrm{B}$ and $\mathrm{E}$. Moreover, the MSD system allows linear measurement through a radial displacement of $11 \mathrm{~mm}$ for magnets $\mathrm{A}$ and $\mathrm{D}$ and of $5 \mathrm{~mm}$ for the others. Finally, the maximum rotation measured by the system is equal to $8^{\circ}$ approximately.

In order to verify the accuracy of the MSD system, careful calibrations have been done to take into account non-linearity of the magnet's response. Calibrations were carried out with a device consisting of a beam on which the magnet is placed, whose displacements are measured by means of two micrometres, respectively in a horizontal and vertical direction. Both have a resolution of 0.001 $\mathrm{mm}$. Starting from the "zero" condition, i.e. alignment of sensors and magnets' centres of gravity, and fixing the position of the magnet in one direction, a displacement in the other direction was imposed with the micrometre. Displacements $\mathrm{z}$ along the vertical direction and displacements $\mathrm{r}$ along the horizontal one were recorded. Results are shown in Fig. 3. The diagrams show a good response of almost all the sensors. However, on average the system records an accuracy of $0.2 \%$ in the vertical direction and $0.3 \%$ in the radial one.
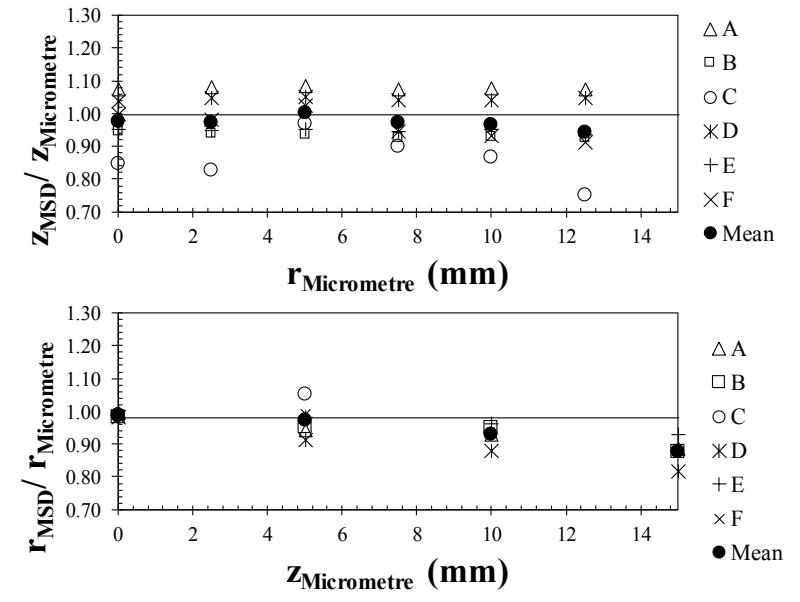

Fig. 3. Calibration tests results on MSD system.

\subsection{Experimental results}

In order to check the performance of the new measurement system MSD, results of an isotropic triaxial tests carried out on a sandy gravel specimen coming from the Coreno pit (Frosinone, Italy (Fig. 4)) are presented below. The tested soil consists in a uniform mixture with particle sizes covering the range $0.85-5 \mathrm{~mm}$ having a specific gravity $\mathrm{G}_{\mathrm{s}}$ of $2.76\left(\mathrm{e}_{\max }=0.83, \mathrm{e}_{\min }=0.61\right)$. The specimens were reconstituted by tamping at an initial relative density $D_{r}=0.70$.

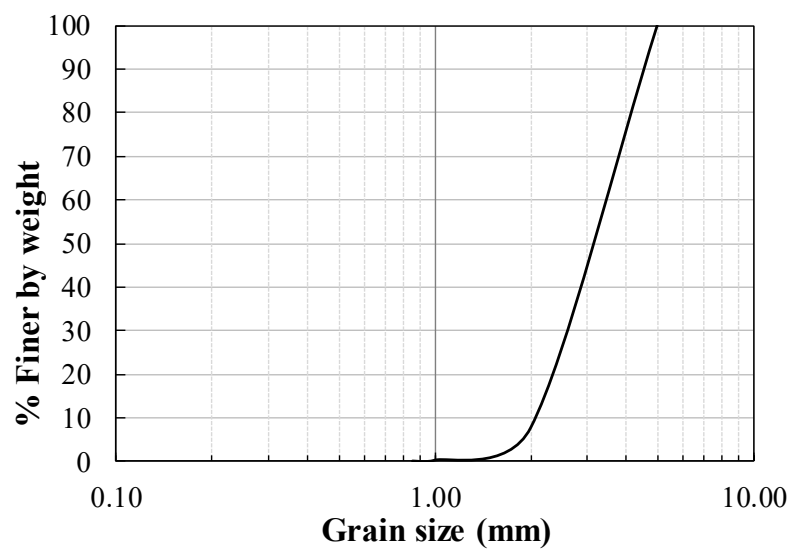

Fig. 4. Grain size distribution of Coreno gravel.

The specimen was saturated and then isotropically compressed up to the maximum value of the mean effective stress p' of $700 \mathrm{kPa}$.

Fig. 5 shows a comparison between the values of the relative axial displacements $(\Delta z)$ of each magnet and the base displacements measured by the LVDT, all normalized with respect to its initial position. Doing so, the LVDT measurement has to be compared with the sum of the MSD readings referring to a single sensor alignment (e.g., $\Delta \mathrm{z}_{\mathrm{LVDT}}=\Delta \mathrm{z}_{\mathrm{F}}+\Delta \mathrm{z}_{\mathrm{D}}+\Delta \mathrm{z}_{\mathrm{B}}=\Delta \mathrm{z}_{\mathrm{C}+} \Delta \mathrm{z}_{\mathrm{A}}+\Delta \mathrm{z}_{\mathrm{E}}$ ). The axial relative displacements measured by the local magnetic system are lower than the ones obtained by the external LVDT transducer, as expected. 


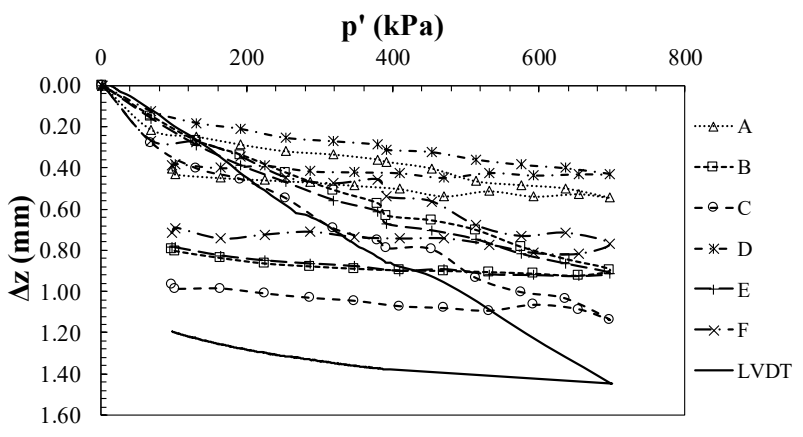

Fig. 5. Relative axial displacements $\Delta z$ versus mean effective stress $\mathrm{p}^{\prime}$ measured by the magnets (MSD) and LVDT transducer.

Fig. 6 shows the elaboration of the mentioned $\Delta z$ in terms of axial strains averaged from the values recorded by all magnets (A, B, C, E and F). The two curves plotted in Fig.6, demonstrate a good agreement between the two measuring systems.

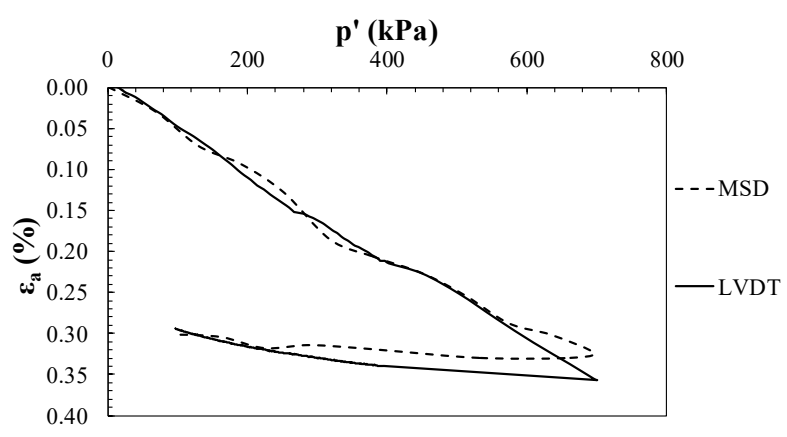

Fig. 6. Comparison in terms of axial strains between internal and external system.

The radial displacements $\Delta \mathrm{r}$ of the specimen are traditionally computed on the basis of the measurement of current volume (by the DPT transducer) and height of the specimen (LVDT data). Fig. 7 compares this kind of measurements with those from the MSD.

It can be observed that there is a large difference between the two kind of measurements: the magnetic system gives a maximum radial displacement equal to approximately a fifth of that one measured by the external system.

The smaller volumetric strain observed with the Magnetic Shape Detector is a clear consequence of magnet's stroke limit in the radial direction and of the greater accuracy of the system in the vertical direction. The first phenomenon was progressively increased as the mean effective stress increased. Therefore, the set up of the magnetic system requires further development operations that minimize the non-linearity errors generated by the complex magnetic field.

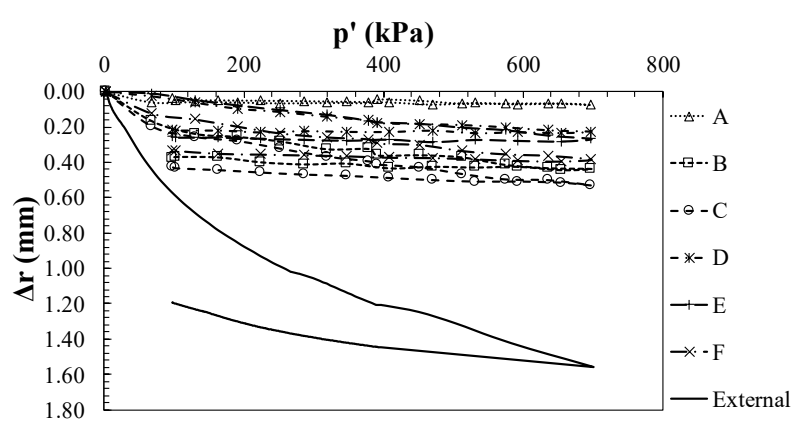

Fig. 7. Radial displacement $\Delta \mathrm{r}$ versus mean effective stress for magnets (MSD) and external measurements.

Clearly, the same difference can be observed in terms of specimen radial strains (Fig. 8).

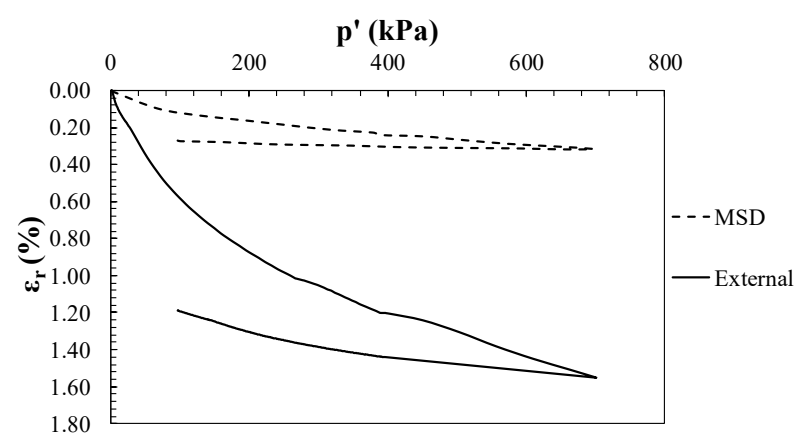

Fig. 8. Comparison in terms of radial strains between internal and external system.

Data shown in Fig. 6 and 8 explain the trend of the curves in Fig. 9. In particular, the External curve represents the DPT transducer measurement instead the MSD curve represents volumetric strains calculated using Eq. 1 with magnetic system data:

$$
\varepsilon_{v}=\varepsilon_{a}+2 \varepsilon_{r}
$$

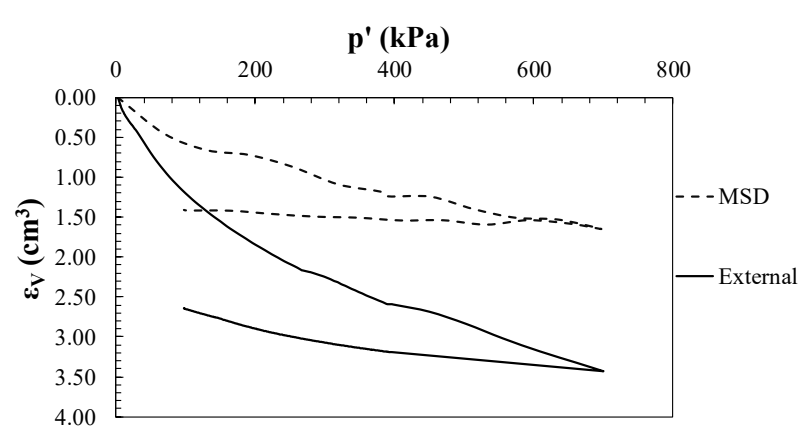

Fig. 9. Volumetric strains $\varepsilon_{\mathrm{v}}$ computed via the processing of the MSD and External system data.

As final aspect, Fig. 10 reports the average shape of the lateral surface of the specimen at fixed time, highlighting the inhomogeneities of the deformation field along the specimen height. The specimen's height was equal to $405.9 \mathrm{~mm}$ and $404.6 \mathrm{~mm}$ at the beginning and at the end of the isotropic compression phase respectively. It is clear that the volume change determination taking into account the non-uniformity of the specimen deformation would better represent the real observed behaviour. 


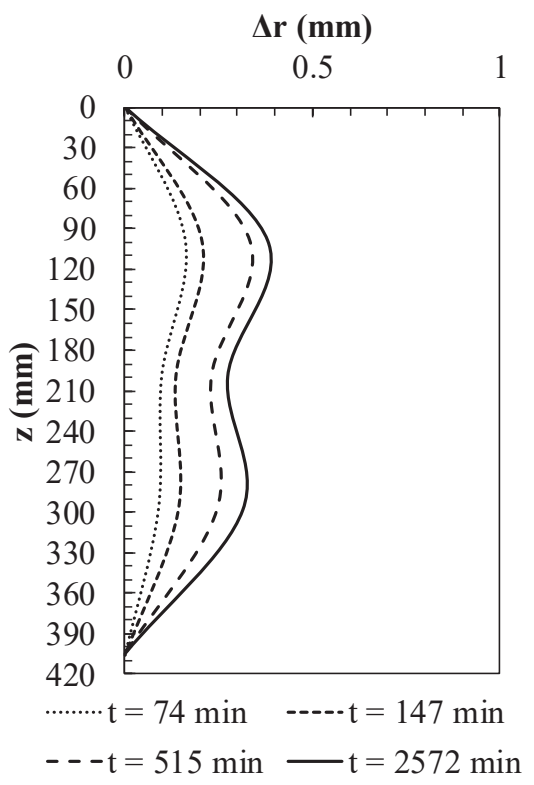

Fig. 10. Progressive development of the lateral profile of the specimen during an isotropic compression test.

\section{Conclusions}

Starting from a pre-existing large triaxial cell HPSP designed to test specimen of coarse soils, auxiliary devices and transducers were set-up to allow the equipment to work also in partially saturated conditions.

In order to monitor local axial and radial strains as well as global volume strains with an adequate resolution in partially saturated conditions, an innovative magnetic shape/volume detector system was developed.

The instrumentations were successfully assessed by performing a series of calibration and triaxial tests in saturated conditions. Calibration tests shown that this local system is an adequate option for measuring axial and radial displacements in large triaxial chambers because the errors are minimal.

However, from the isotropic compression test carried out on a sandy gravel with a maximum particle size of 5 $\mathrm{mm}$, the magnetic system appears to be greatly affected by a non-linear response of the magnet through a large displacement range in the radial direction. Instead it was applied satisfactorily to measure the axial strain in the specimen. The volumetric strains measurement method based on the magnetic system was proved to be effective for the determination of the volume during triaxial tests, but it requires further setting up operations.

\section{References}

1. L. A. Oldecop, Phd thesis, Universitat Politècnica de Catalunya, Spain, (2000)

2. E.E. Alonso, A. Gens, A. Josa, Geotech., 22, 3, 405430 (1990)

3. L. A. Oldecop, E. E. Alonso, Geotech., 52, 2, 127139 (2001)

4. L. A. Oldecop, E. E. Alonso, Geotech. Test. J., 27, 3, 1-10 (2001)
5. A. W. Bishop, L. D. Wesley, Geotech., 25, 4, 657670 (1975)

6. E. Romero, J. A. Facio, A. Lloret, A. Gens, E. E. Alonso, 4th ICSMFE Hamburg, 1, 185-188 (1997)

7. S. Messerklinger, E. Bleiker, Zweidler A., S.M. Springman, 16th European Young Geothecnical Engineers' Conference, 1-10, Vienna, Austria. (2004)

8. J.M. Camacho, V. Sosa, Revista Mexicana de Fisica, 59, 8-17 (2013) 